\title{
Nucleation and Structural Identification in Gold Particles of High Aspect Ratios Developed through Mechanistic Approach
}

\author{
Mubarak Ali ${ }^{\text {* }}$ and I -Nan Lin ${ }^{b}$ \\ aDepartment of Physics, COMSATS University Islamabad, Park Road, Islamabad - \\ 45550, Pakistan, `E-mail: mubarak74@mail.com, mubarak74@comsats.edu.pk \\ bDepartment of Physics, Tamkang University, Tamsui Dist., New Taipei City 25137, \\ Taiwan (R.O.C.). E-mail: inanlin@mail.tku.edu.tw
}

\begin{abstract}
A structural identification in different geometrical shapes of gold particles is presented here. Nucleation mechanisms of particles having geometrical shapes are discussed here, which have never been reported before. Dimensional regularity of particles in geometrical shapes incites a new insight. At electronically flat solution surface, different zones have been found dealing with the developing tiny-shaped particles in less elongation of atoms and more elongation of atoms. Tiny-shaped particles in less elongation of atoms nucleate particles of one-dimensional (1D) shapes due to developing in a zone consisting of regions rearward to north-pole at solution surface. Tiny-shaped particles in more elongation of atoms nucleate multi-dimensional (MD) shapes due to developing in a zone consisting of east-west regions at solution surface. To assemble at a common point forming at the centre of concave meniscus, structures of smooth elements at electronically decreasing level solution surface experience force in immersing format. A force exerting in the immersing format is related to the simultaneous actions of four forces to a structure of smooth element coming to assemble. In addition to the acquired orientation of an electron and the position of its atom at solution surface, a manner of energy knot clamping to electron in an atom also varies exertion of force for it. Particles of geometrical shapes show different structures in 1D and MD shapes. On identifying structure, a mechanism of photon reversion is disclosed. In the selected area patterns of particles, printing spots of reverted force in photons reflected from the laterally orientated electrons of less and
\end{abstract}


more elongated atoms validates that photons are not carried by the electrons, so it is a photon reflection instead of an electron diffraction.

Keywords: Atomic behavior; Elongation; Fundamental forces; Adjacent \& Lateral orientations; Nucleation \& Structure; Photon interaction

\section{Introduction}

Understanding the atomic behavior with new insight is an integral part of physical and chemical sciences. Atomic world is unique but diverse, too. Atoms of different elements do not incite understanding based only on their physical behaviors, but interactions with various counterparts can also be considered. The study of inclined planes is familiar to us when studying science at basic level. The study of inclined plane helps to solve fundamental problems of Mathematics, which is not only limited to the equation of slope intercept form, but also to other trigonometric formulations. However, an inclined plane can be particularized when the force at electron level in elongated atoms of arrays is exerted in the immersing format. Such arrays can be sorted at the centre of concave meniscus formed by electronically decreasing level solution surface all around the solution surface.

Atomic structure of different state carbon atoms along with the conversion mechanism for different states is discussed by Ali [1]. Incompatible working energy and forced behaviors of gas and solid atoms are explained in developing the structure of hard coating [2]. As per ground points of atoms when they are in neutral states, they evolve different structures depending on the generated binding energy under confined inter-state electron dynamics [3]. Atoms of none of the elements ionize [4]. Under the neutral state of silicon atom, heat energy converts into photon energy, where confined inter-state electron dynamics execute [5]. The origin of atoms in some elements to be a gas and in some elements to be a solid is explored [6]. Depending on the mode of electron dynamics of atoms, different outcomes of their developed tiny-sized particles can be anticipated [7]. The fundamental process of developing different tiny-sized particles depends on the effects of electronic structure, phase transition and localized dynamics of atoms [8]. Different precursors develop different shapes of tiny-sized particles when their solutions are processed even for the same conditions of process 
[9]. In the formation of tiny particles and their extended shapes, different orientations of electrons under varied potential energy in their atoms were discussed [10]. Different pulse ON/OFF times release different shapes of nano-energy to bind transitional behavior atoms at electronically flat solution surface [11]. Many tiny-sized particles developed in equilateral triangular shape when suitable concentration of gold precursor was processed [12]. A detailed study of developing tiny-shaped particle and modifying atoms of arrays into structures of smooth elements is discussed elsewhere [13]. The origin of functioning in-situ predictor packing for developing highly anisotropic shapes of gold particles has been explored by Ali et al. [14].

A force exerting along the south-pole of electrons in (solid) atoms of various elements can be considered entering from the north-pole. The reverting of the element of force when featured photon interacts with suitably arranged clamped electron of atom can be considered the source of levitational force functioning at the certain level of ground surface. So, a (stored) force functioning in the grounded format can be considered entering by leaving the certain level of ground surface.

When atoms of suitable elements under a certain transition state reach the level of exerting force in the surface format, they can also be considered eligible for shaping tiny-sized particles. The study has discussed developmental mechanism of tiny particles in such atoms when the packets of nano-energy were supplied [13]. Our several experimental studies have discussed binding of transitional behavior gold atoms in a triangular shape under optimized pulse ON/OFF times [9-12]. Thus, a tiny particle of mono layer develops when transitional behavior atoms of 'compact monolayer assembly' rest at electronically flat solution surface and is empowered through the protocol energy called nano-energy. However, in case where atoms do not deal with the resting positions at electronically flat solution surface, their binding in different tiny particles is more influenced by the deformed behavior instead of elongated behavior [4].

When tiny particles of triangular shape also develop from atoms of monolayer assembly at the level, where force in surface format is exerted, arrays of atoms are converted into structures of smooth elements [13]. Hence, structures of smooth elements belonging to each tiny-shaped particle function as one unit while assembling at the common point. At electronically decreasing level solution surface, an exceeded 
level of exerting force to electrons of forwarding sides of 'structures of smooth elements' needs to be considered in the immersing format. To develop colloidal particles of a choice, a concave meniscus of choice can be developed at solution surface under the entrance of photons and electrons through $\mathrm{Cu}$ capillary [8-12]. This way, a point of assembling structures of smooth elements can be made at point of choice instead of at the centre of solution surface. This can work out for different features of tiny particles, nanoparticles and particles.

Some recent studies have discussed the functionalities of nanomaterials observed under high resolution microscopic images [15, 16]. At different concentration of gold atoms, different sized hexagonal-close packed structures emerged [17], and the role of dynamics in different sorts of structural formation is considered crucial [18-22]. When nanoparticles are synthesized in the solution, they provide understanding of the structure at nanoscale [23]. Jacobsson et al. [24] observed the change in crystal phase by varying the growth conditions. The physics of switching amorphous phase to crystal phase is explored [25], and phase transition in vanadium dioxide results in changing the optical properties [26]. The understandable assembling of tiny-metallic colloids into large metallic colloids enables the treatment of atoms and molecules as materials of tomorrow [27]. It is necessary to first investigate dynamics of developing small-sized particles prior to understanding the developing mechanisms of large-sized particle [28].

The forced exertions to electrons of gold atoms developing tiny particles of specific shape in different regions of two categorized zones of solution surface are not yet clear. When arrays of gold atoms modify into structures of smooth elements, they assemble at a common point to nucleate particles of geometrical shapes. Therefore, the nucleation mechanism and identifying precise structures in particles of geometrical shapes are the key to explore. The forced exertions to nanoscale components and atoms standalone are expected to play role in different ways. The forced exertions to electrons of elongated atoms at electronically flat solution surface and at electronically decreasing level solution surface are also expected to contribute in a different way. A detailed investigation on structural analyses of particles of 1D and multi-dimensional (MD) shapes is also discussed in this study. A different elongation rate of atoms in the 
particles of $1 \mathrm{D}$ and MD shapes is identified under the application of featured photons inferring a photon reflection instead of an electron diffraction.

\section{Experimental details}

Experimental details of synthesizing different colloidal particles along with layout of the setup are given elsewhere [14]. Again, preliminary studies on the developmental mechanism of such particles along with their syntheses have been discussed [9-14]. At solution surface, the centre of the concave meniscus was developed under the midpoint of light-glow known in plasma spot, where different particles of 1D and MD shapes developed one by one. The key parameters to attain the optimized conditions for developing particles of 1D and MD shapes are given in the Figures' captions. Particles of gold discussed here for nucleation and analysis (of structure) were actually developed in the centre of light-glow formed at solution surface, which is related to the centre of concave meniscus. The light-glow is appeared on entering forcing energy of travelling photons and electrons of carrying forcing energy (of chasing photons) inside the solution. The particles exhibiting dominant features are discussed in this study. Their selected area photon reflection patterns were captured to understand the formation of precise structure. Both geometry and the structure of selected particles are discussed here. Hence, this study does not focus on the methodological process of developing the particles of different geometrical shapes. Instead, it focuses on the nucleation mechanism of rod and plate-shaped particles. Analyses of the particles from the selected area identify structural features exploring a mechanism of photon reflection, too.

\section{Results and Discussion}

Particles having 1D shape (rod and bar) are shown in Figures 1 (a, b, c, d, e, f \& g). Particles developed in different aspect ratios. The approximately measured aspect ratios (ratio of longer length to width) of particles are $-61: 1,30: 1,21: 1,54: 1,20: 1,33: 1$ and 35:1. They are shown by the bright field transmission microscope (BF-TM) images in Figure 1 (a), (b), (c), (d), (e), (f) \& (g) respectively. The lowest aspect ratio of the particle is shown in Figure 1 (e), whereas the highest aspect ratio of the particle is 
shown in Figure 1 (a). Different particles of shapes such as rod and bar printed intensity spots in the form of lines having their regular order in respective selective area photon reflection (SAPR) patterns are shown in Figure 1 (A, B, C, D, E, F \& G). However, due to the packing of tiny-shaped particles and the assembling of their structures of smooth elements at low degree angles, SAPR pattern of each 1D shape indicates printed intensity spots in the shape of fixed distant parallel lines. In different SAPR patterns of particles with shape such as rod and bar, a projected normal line to any of the printed line of intensity spots gives an approx. angle of assembling of structures of smooth elements. Here, low degree angles specify the assembling of structures of smooth elements (in the development of 1D shapes of particles or nanoparticles) at smaller degree orientations, i.e., $\sim 5^{\circ}$ to $\sim 25^{\circ}$.

a

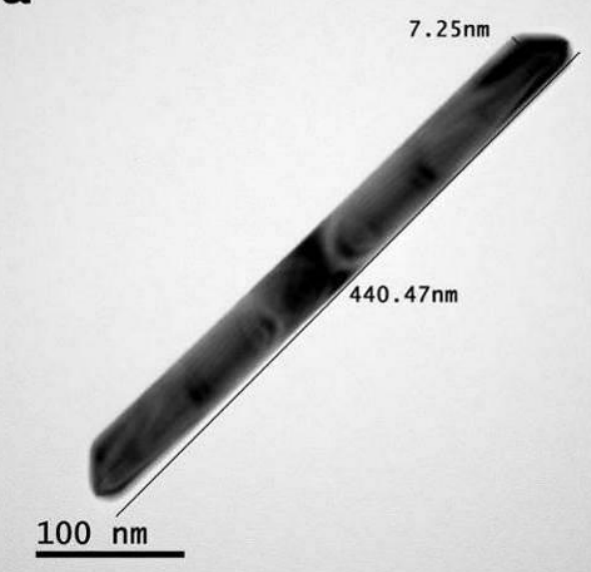

b

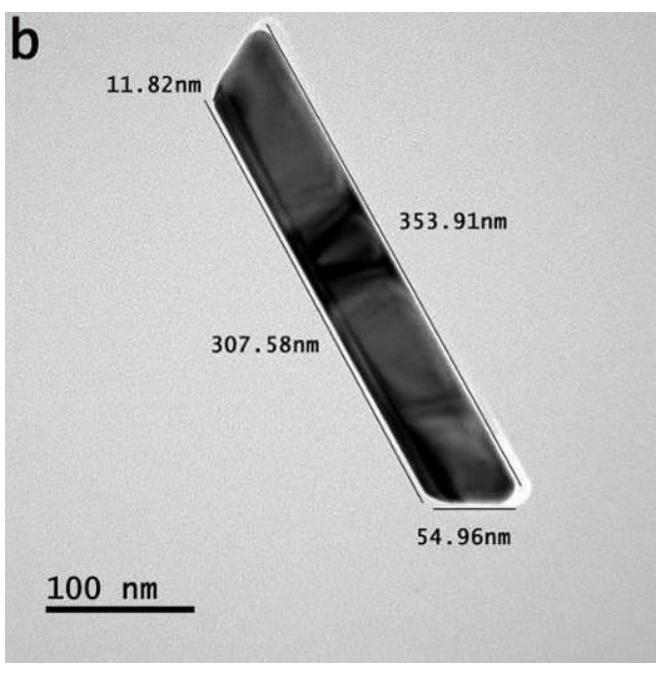

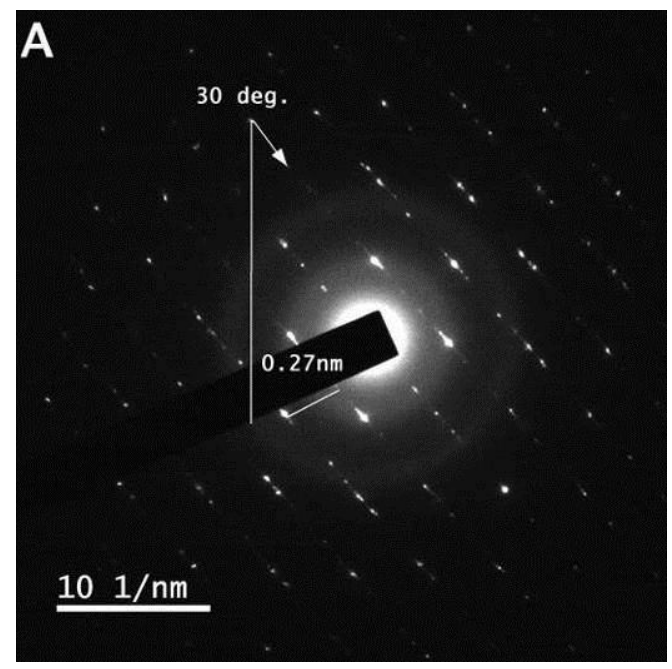

B

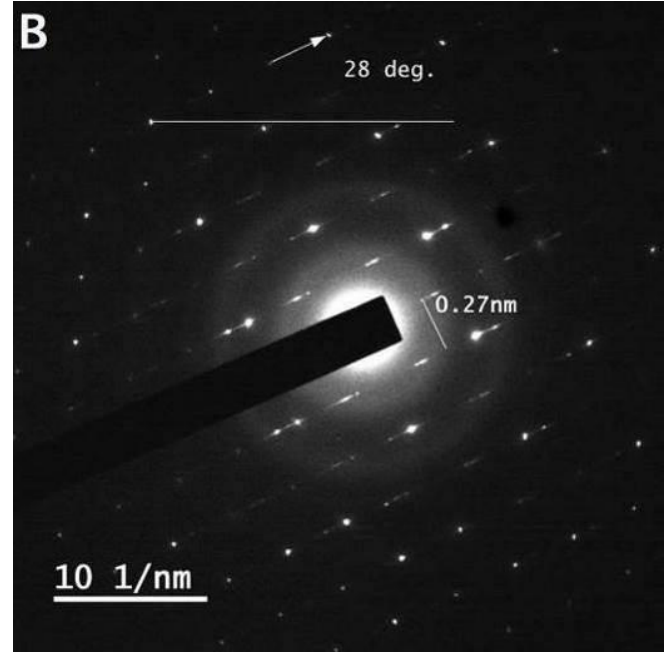


C

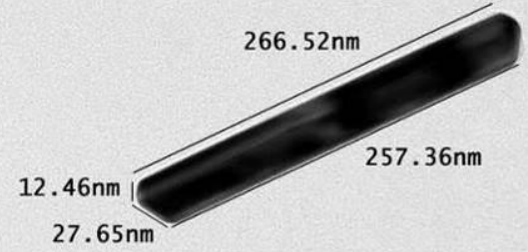

$100 \mathrm{~nm}$

d

$100 \mathrm{~nm}$

e

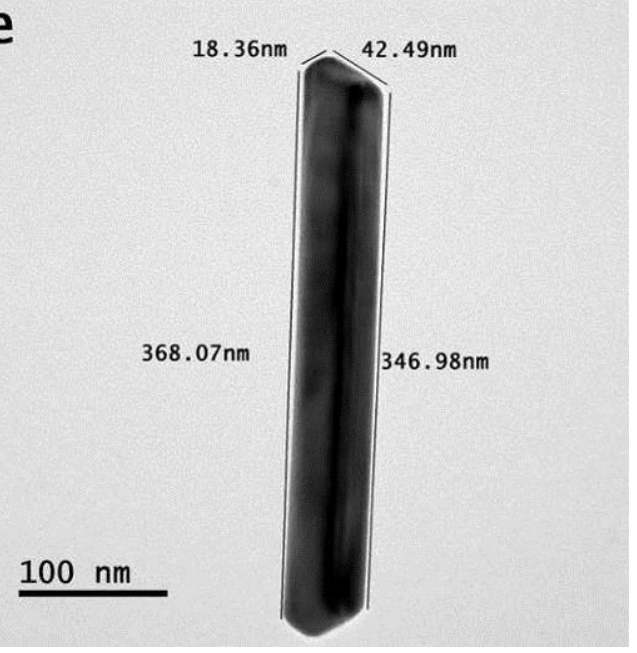

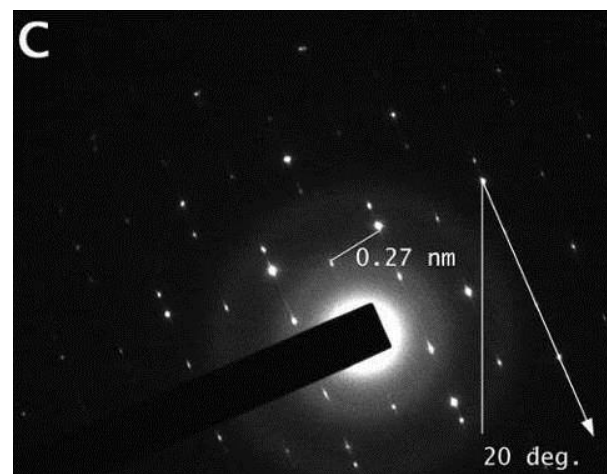

$10 \quad 1 / \mathrm{nm}$
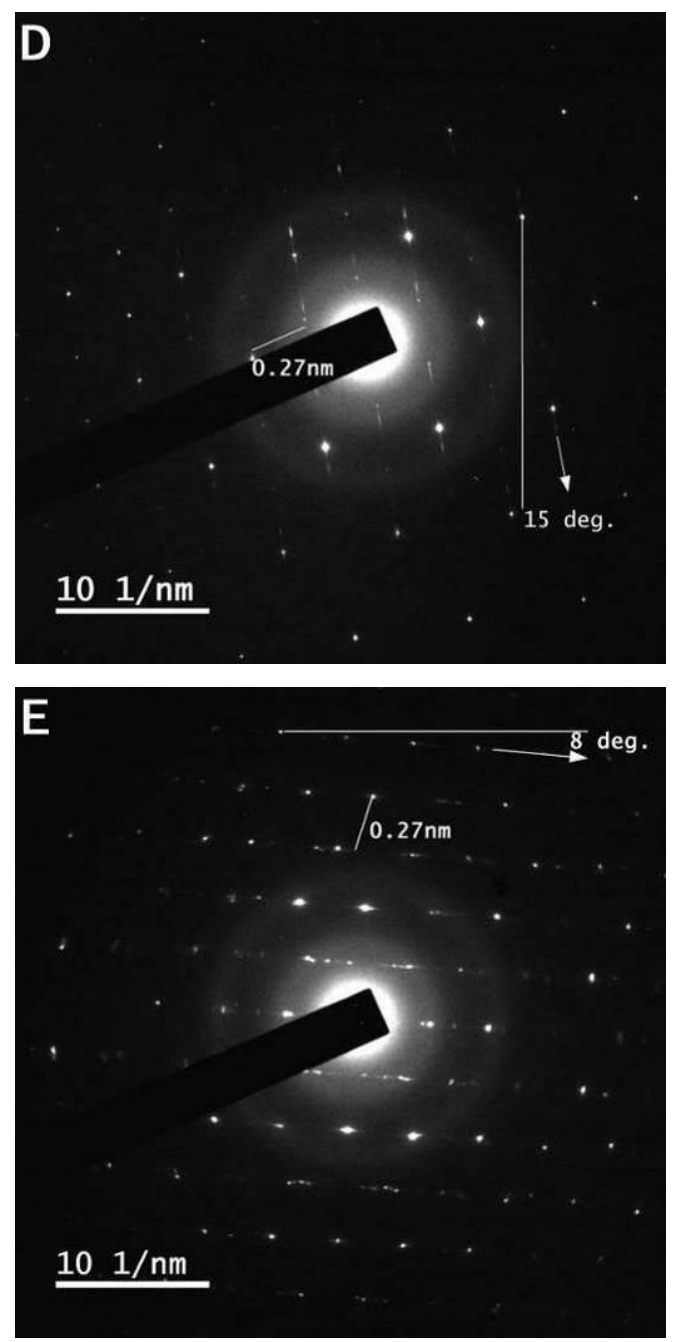

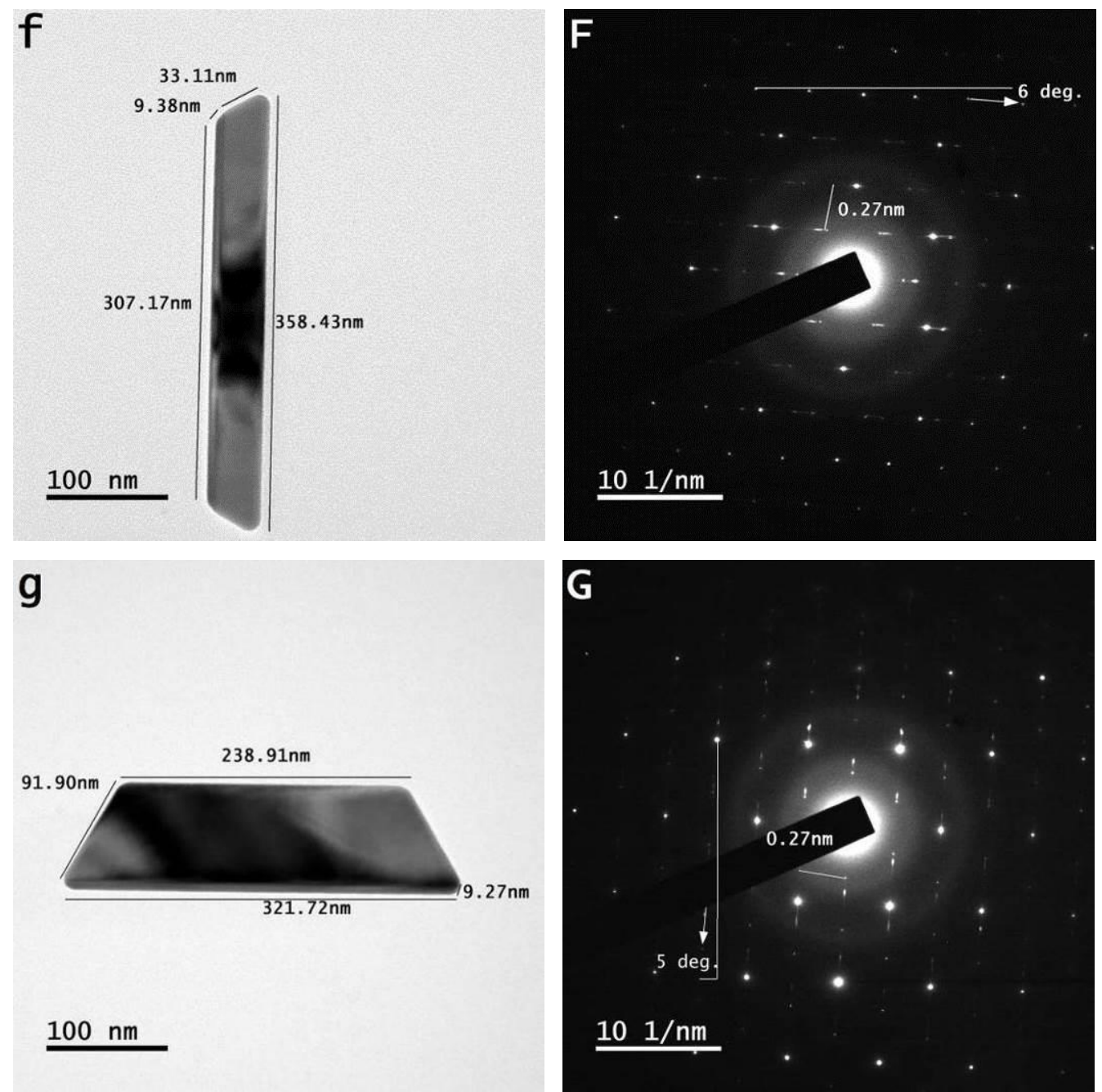

Figure 1: $(a, b, c, d, e, f \& g)$ BF-TM images of different aspect ratios gold particles of 1D shapes and their respective SAPR patterns (A, B, C, D, E, F \& G); pulse ON/OFF time: $10 \mu$ sec, concentration: 0.30 $\mathrm{mM}$ and process duration: 20 minutes

For each high aspect ratio particle of 1D shape, structures of smooth elements in each mono layer indicate uniform widths. In Figure 1 ( $A, B, C, D, E, F$ \& G), an approximately measured length of centre to centre distance of two parallel lines of printed intensity spots is $\sim 0.27 \mathrm{~nm}$. SAPR patterns of various particles of $1 \mathrm{D}$ shapes show precise assembling of structures of smooth elements, where tiny-shaped particles are packed as one unit to develop their mono layers. In those particles, not only the electrons of zeroth rings of elongated atoms (forming structures of smooth elements) are aligned laterally, but certain electrons of their outer rings are also aligned laterally. Reflected photons from the selected areas of particles having 1D shapes spotted (printed) intensity spots in patterns are in the shape of a line. Printed intensity spots in 
the shapes of lines are parallel to each other, where inter-spacing distance is fixed. However, atomic structures in different elements describe both zeroth rings and outer rings [1-6], which were previously known in shells and orbits.

Different particles of MD shapes having different aspect ratios are displayed in Figure 2 ( $a, b, c, d \& e)$, where respective SAPR patterns are shown in Figure 2 (A, B, $C, D \& E)$. Different particles import packing of tiny-shaped particles from different regions of solution surface and assembling of structures of smooth elements are under their controlled orientations. However, certain thermodynamic driving forces are required to be considered here. In different SAPR patterns shown in Figure 2 ( $A, B, C$, $D \& E$ ), the approx. measured inter-spacing distance of intensity spots is $\sim 0.24 \mathrm{~nm}$ (centre to centre), which is found to be the same for each face (dimension) of the particle. In SAPR pattern of each particle having MD shape, front side area from any face (dimension) is being analyzed. Each structure of smooth element is in parallel arrangement to keep the mono layer. In different SAPR patterns of particles having MD shapes, distance (centre to centre) between any two nearby circular dots or intensity spots is $\sim 0.12 \mathrm{~nm}$. A width belonging to a structure of smooth element is $\sim 0.12 \mathrm{~nm}$. Hence, their total distance is $\sim 0.24 \mathrm{~nm}$.
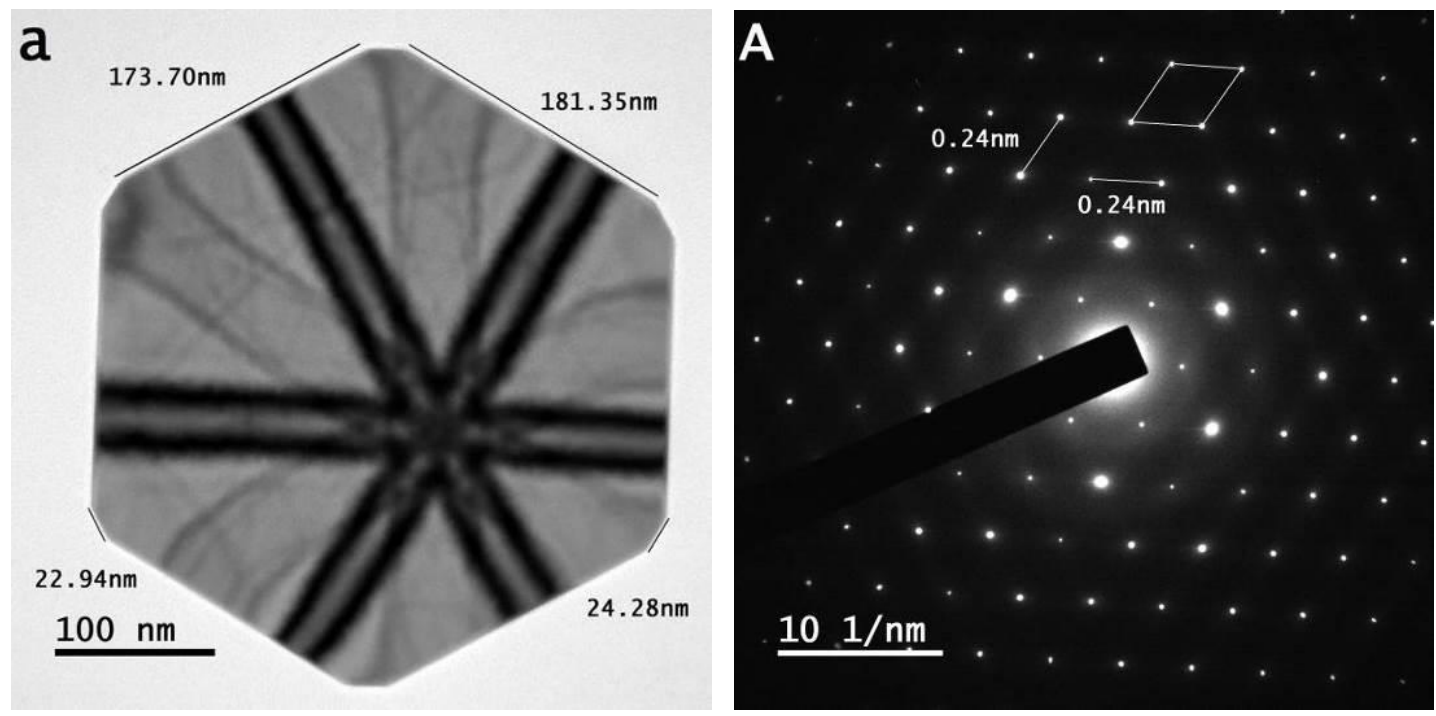

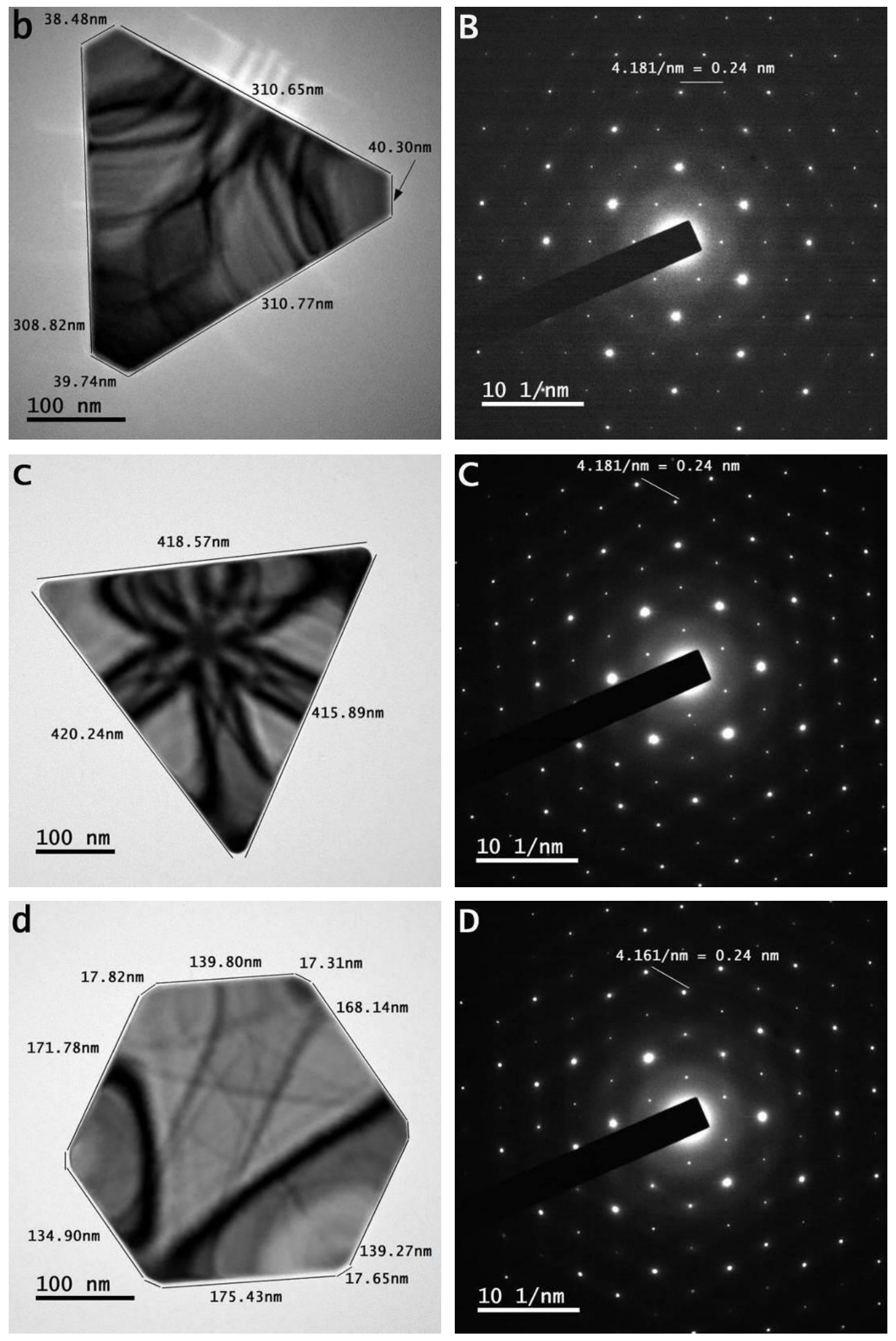

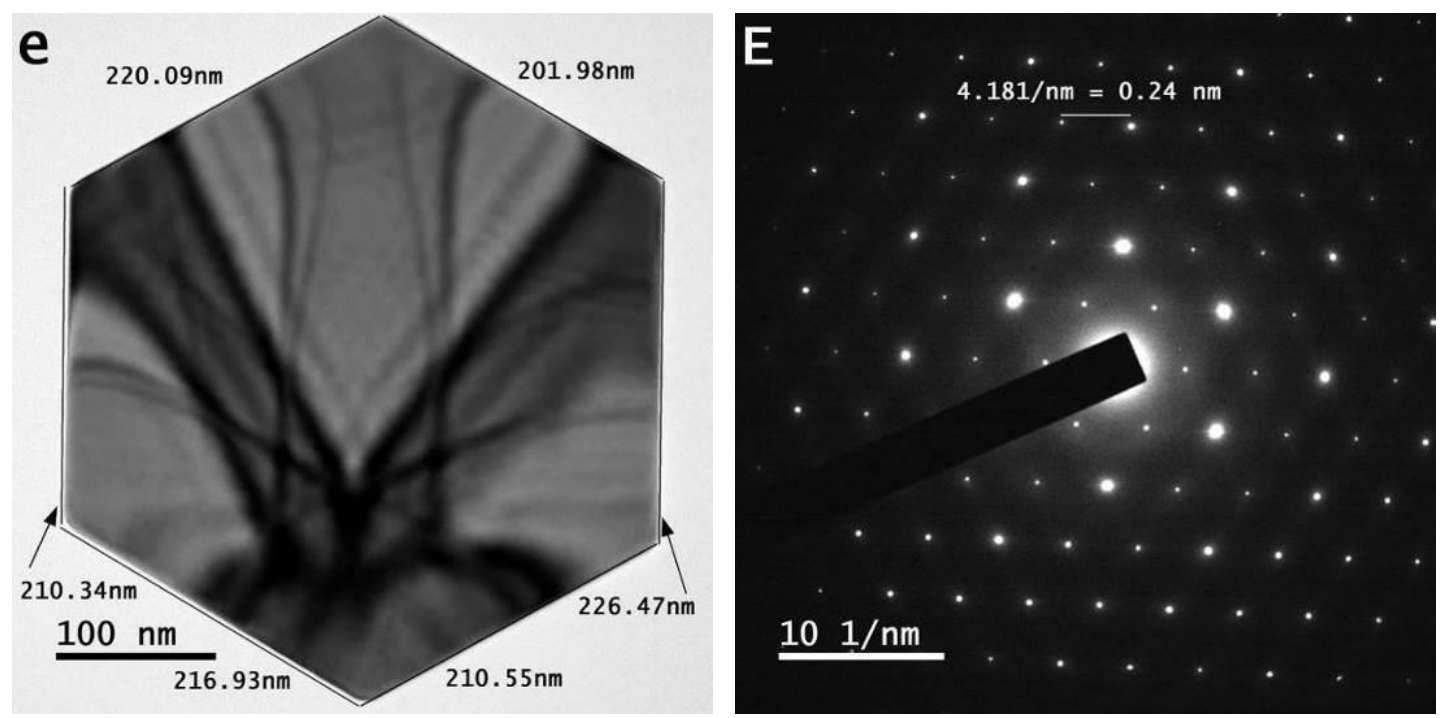

Figure 2: (a, b, c, d \& e) BF-TM images of different aspect ratios particles of MD shapes and their respective SAPR patterns ( $A, B, C, D \& E$ ); bipolar pulse ON/OFF time: $10 \mu \mathrm{sec}$, precursor concentration: $0.30 \mathrm{mM}$ and process duration: 20 minutes

Particles of MD shapes are related to assembling of structures of smooth elements (of tiny-shaped particles) at high degree angles. However, tiny-shaped particles come from different regions of the solution surface having nearly identical nature of the exerting force at electron level. This is not the case in developing particles of 1D shape, where assembling of structures of smooth elements (of tiny-shaped particles) are at low degree angles experiencing force at lower influence and at smaller degree orientations. This way, their tiny-shaped particles come from different regions of solution surface. Printed intensity spots of reflected photons in the patterns of particles having MD shapes are in the form of dots; upper two dots are from the upper two elongated atoms (forming a structure of smooth element) and lower two dots are from the lower two elongated atoms (forming a structure of smooth element) as shown in the respective SAPR patterns. Dot to dot distance is the same in SAPR patterns of MD shapes. Centre to centre distance between any two nearby dots is also the same. Here, high degree angles specify the assembling of structures of smooth elements at larger degree orientations $\left(\sim 30^{\circ}\right.$ to $\left.\sim 72^{\circ}\right)$ as in the case of MD shapes of nanoparticles or particles.

In SAPR patterns of particles having MD shapes, intensities of reflected photons print the shape of circular dots in repeating order with a fixed centre to centre distance. 
However, in case of a particle having 1D shape, each structure of smooth element has more width, where not only electrons belonging to zeroth rings of elongated atoms remained in lateral orientation, but some of the electrons of outer rings also remained in lateral orientation. Thus in the SAPR patterns of particles having rod or bar shapes, intensities of reflected photons are in the shape of lines. However, parallel lines have fixed mid to mid distance, so electron diffraction is a photon reflection.

In Figure 3 (a), a pentagon-shaped particle is shown in the image captured by high resolution transmission microscope (HR-TM). A region covered under the square box is further magnified in Figure 3 (b). Here, the approx. measured width of each structure of smooth element is $0.12 \mathrm{~nm}$. Mid to mid measured distance between any two structures of smooth elements is $\sim 0.24 \mathrm{~nm}$ as width of each structure of smooth element is $\sim 0.12 \mathrm{~nm}$ as shown in Figure 3 (b); inter-spacing distance among structures of smooth elements is uniform, i.e., $\sim 0.12 \mathrm{~nm}$. In Figure 2 (A, B, C, D \& E), SAPR patterns of particles of MD shapes also show the centre to centre distance of two dots, i.e., $\sim 0.24 \mathrm{~nm}$.
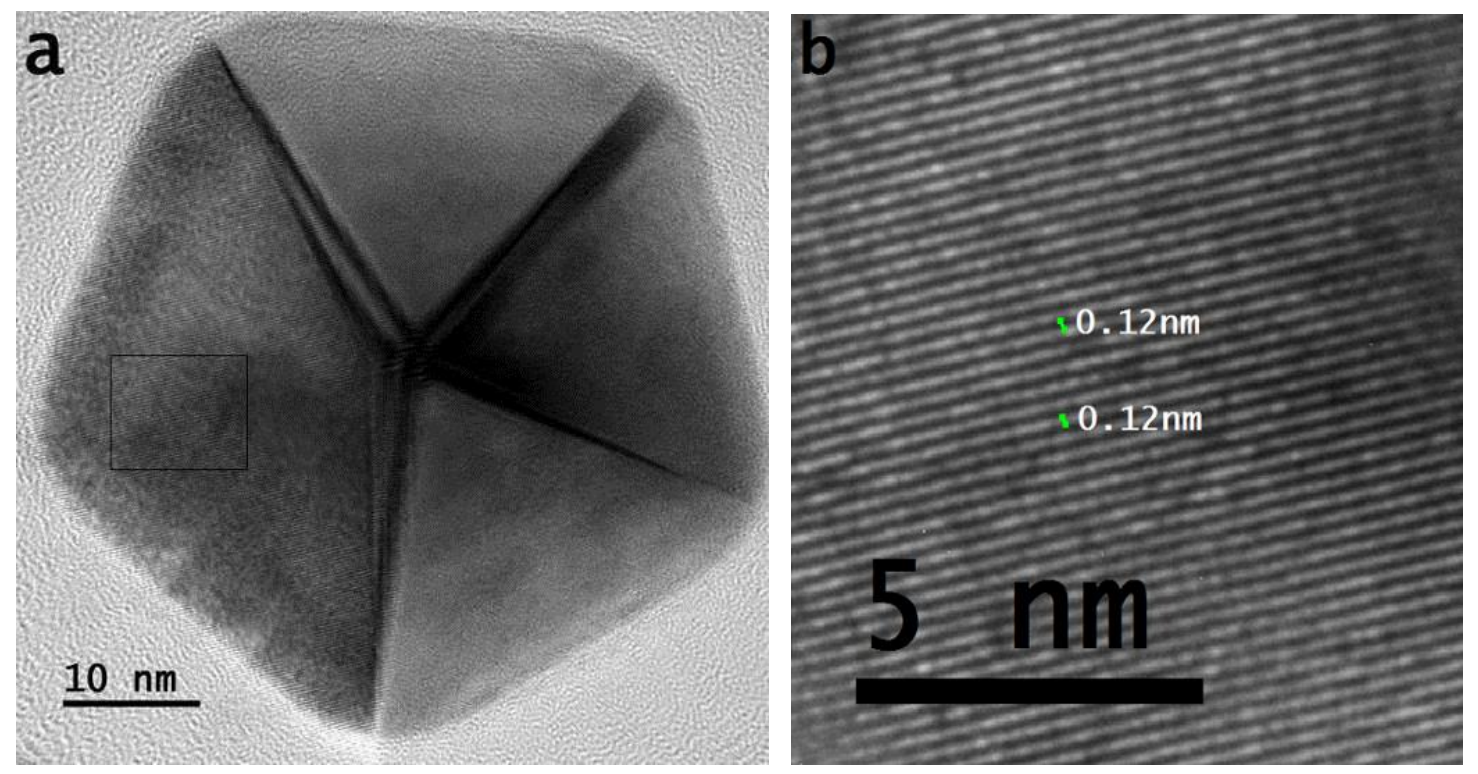

Figure 3: (a) HR-TM image of pentagon-shaped nanoparticle and (b) magnified image of marked region in square box of Figure 3 (a) shows equal inter-spacing distance among structures of smooth elements $(\sim 0.12 \mathrm{~nm})$ and an equal width of each structure of smooth element $(\sim 0.12 \mathrm{~nm})$; bipolar pulse ON/OFF time: $10 \mu \mathrm{sec}$, precursor concentration: $0.30 \mathrm{mM}$ and process duration: 2 minutes 
In structures of smooth elements, electrons of outer rings in atoms do not align in lateral orientation. Electrons of zeroth rings in elongated atoms mainly align in lateral orientation. Thus, featured photons interact with north-sided tips of electrons laterally orientated in clamping energy knots belonging to zeroth rings of elongated atoms. On reflection, they resulted in reverting the element of their force. So, they spot (print) intensity in the form of circular dots in the pattern. Therefore, the distance between dots of spotted intensity spots also becomes equal as it is in SAPR patterns of particles shown in Figure 2 ( $A, B, C, D \& E$ ). A measured centre to centre distance of two dots from either side in patterns of particles having MD shapes is the same as measured in the case of HR-TM image of particle having MD shape shown in Figure 3(b).

A structure of smooth element is related to elongated atoms of an array, where the exerting forces to electrons of outer rings orientate them adjacently [13]. In different particles of MD shapes, structures of smooth elements develop on elongating atoms of arrays (of tiny-shaped particles). Their measured width is less. Mainly electrons of zeroth rings in elongated atoms remained aligned along orientational orientation. Therefore, reflected featured photons spotted (printed) intensity spots (instead of intensity lines) in the resultant pattern of particle of MD shape. In elongated atoms of $M D$ shapes, reflected photons interacted laterally orientated electrons of zeroth rings mainly, so the resulted inter-spacing distance of printed intensity spots in particles of MD shapes are slightly less as compared to particles having 1D shapes.

In developing a particle of geometrical shape, tiny-shaped particles only pack for regions of its vacant (unfilled) sites. Prior to the packing of tiny-shaped particles, atoms of each array elongate and modify into a structure of smooth element (at electronically flat solution surface). Each structure of smooth element preserves its features in the tiny-shaped particle. In particles of 1D shapes, distance between two structures of smooth elements can be measured $\sim 0.14 \mathrm{~nm}(0.27 \mathrm{~nm} / 2=0.135 \mathrm{~nm})$. In this way, a width of structure of smooth element also becomes $\sim 0.14 \mathrm{~nm}$. This is also obvious in SAPR patterns shown in Figure 1 ( $A, B, C, D, E, F \& G$ ). In particles of MD shapes, distance between two structures of smooth elements can be measured $\sim 0.12 \mathrm{~nm}(0.24$ $\mathrm{nm} / 2=0.12 \mathrm{~nm}$ ). In this way, a width of structure of smooth element also becomes $\sim 0.12 \mathrm{~nm}$. This is also obvious in SAPR patterns shown in Figure 2 (A, B, C, D \& E). 
Different tiny-shaped particles developed under the supply of packets of nanoenergy [11]. Packets of tuned bipolar pulses (ON/OFF time: $10 \mu \mathrm{sec}$ ) are in controlled behavior of force and energy forming the shape of joined triangles. So, packets of nanoenergy over compact monolayer assembly of gold atoms isolate them, where atoms shape in tiny particles in shapes such as joined triangles in each case. Because of a bit perturbed exertion of force at electron level in face to face joined atoms, a block of joined triangular shape tiny particles is separated into two equal triangle-shaped tiny particles [13]. However, triangle-shaped tiny particles also get developed directly under a unipolar mode of tuned pulse ON/OFF time [11].

In developing structures of smooth elements of a tiny-shaped particle, atoms align electrons (of outer rings) adjacently by keeping them clamped in energy knots. To nucleate each mono layer of particles of 1D and MD shapes, tiny-shaped particles develop into the structures of smooth elements in a similar manner. However, at nucleation stage of particles of $1 \mathrm{D}$ and MD shapes, tiny-shaped particles come from the different regions of two categorized zones at solution surface. As the atoms of gold element under certain transition state rest at electronically flat solution surface in the form of monolayer assembly, they develop tiny particles in the shape of nano-energy supplied by the tuned bipolar pulses. At solution surface, elongation rate of atoms forming tiny-shaped particles in a zone consisting of east-west regions (and east-west near regions) is greater than that of the atoms forming tiny-shaped particles in a zone consisting of regions rearward to north-pole (and near regions rearward to north-pole). Therefore, arrays of elongated atoms of tiny-shaped particles in two different zones keep different widths of structures of smooth elements; in particles of MD shapes, width is $\sim 0.12 \mathrm{~nm}$ and in particles of ID shapes, width is $\sim 0.14 \mathrm{~nm}$.

In this study, when it is said, "Less elongated atoms forming structures of smooth elements" as in the case of rod or bar-shaped particles, it is due to less orientated (adjacent-wise) position of electrons. The energy knots clamped to electrons stretched along the same axis to a less extent but in a controlled manner. Moreover, when it is said, "More elongated atoms forming structures of smooth elements" as in the case of triangle or hexagon-shaped particles, it is due to more orientated (adjacent-wise) 
position of electrons. The clamped energy knots to electrons stretch along the same axis to a greater extent but in a controlled manner.

In the adjacent-wise assembling process of two structures of smooth elements for developing rod or bar shaped particle, electron (belonging to outer ring of an elongated atom) at the tip of one structure of smooth element undertakes another clamp of energy knot clamping to unfilled state (belonging to outer ring of an elongated atom) at the tip of other structure of smooth element. Simultaneously, these structures of smooth elements also undertake double clamping of electron under opposite direction of mechanism. So, two structures of smooth elements assemble while exerting the forces along the opposite poles of electrons, where binding is by means of electrons of filled states and energy knots of unfilled states (of elongated atoms) available at their tips. Structures of smooth elements as one unit form the shape of tiny particle like a triangle. They experience force in immersing format at electronically decreasing level solution surface.

Electronically decreasing level solution surface is hypothetically shown in Figure 4 (a), where the simultaneous actions of forces assemble each structure of smooth element at a common point. A common point is formed at the centre of concave meniscus, which is also a midpoint of light-glow (at solution surface) in the process of synthesized particles shown in Figures 1 to 3 . Structures of smooth elements assemble at a common point when they experience force in immersing format. Forces exerting in immersing format means that they function for structures of smooth elements at electronically decreasing level solution surface. Structures of smooth elements remain at solution surface while adhering their shaped mono layer to (structures of smooth elements of) underneath shaped mono layer.

An increased surface force is exerted along the assembling ends and a decreased surface force is exerted along the non-assembling ends of structures of smooth elements when their tiny-shaped particles come from different regions of categorized zones (two) at solution surface. In immersing format, the exerting force to structure of smooth element along assembling end becomes greater than the exerting force along non-assembling end, where an exerting force in space and grounded formats competes for equivalence. So, electronically decreasing level solution surface 
deals with exertion of forces in immersing format instead of exertion of forces in single format, such as space format, surface format or grounded format. These are shown hypothetically in Figure 4 (b). Forces exerting in space format and grounded format become equal at the centre of concave meniscus. Forces exerting along the assembling ends and non-assembling ends of structures of smooth elements also become equal at the centre of concave meniscus. So, structures of smooth elements of first developed shaped mono layer remain in the centre of concave meniscus at solution surface till the positioning of another similar shape and size shaped mono layer above.

(a) Assembling structures of smooth elements at common point (the centre of concave meniscus at midpoint of light-glow)

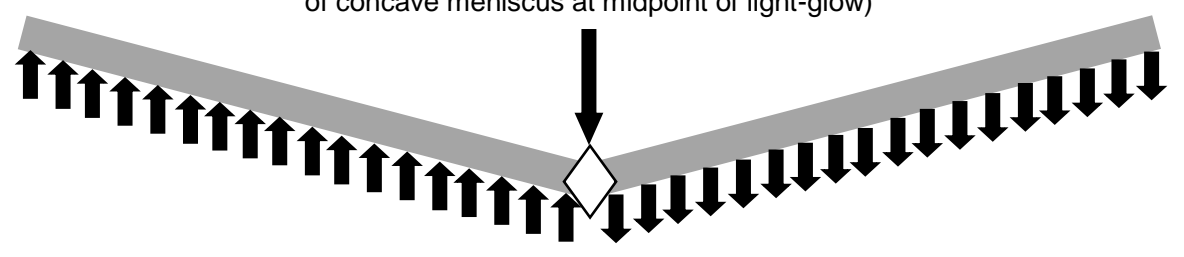

(b) (1)

Electronically decreasing level solution surface

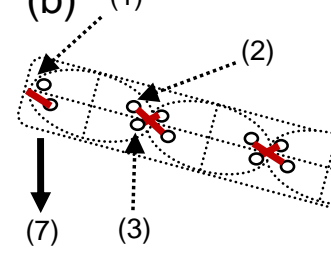

(c)

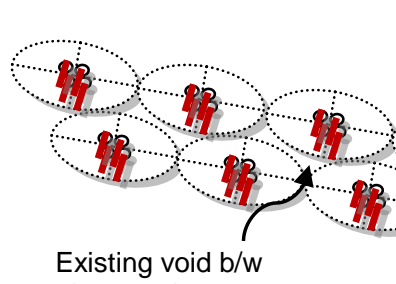

elongated atoms

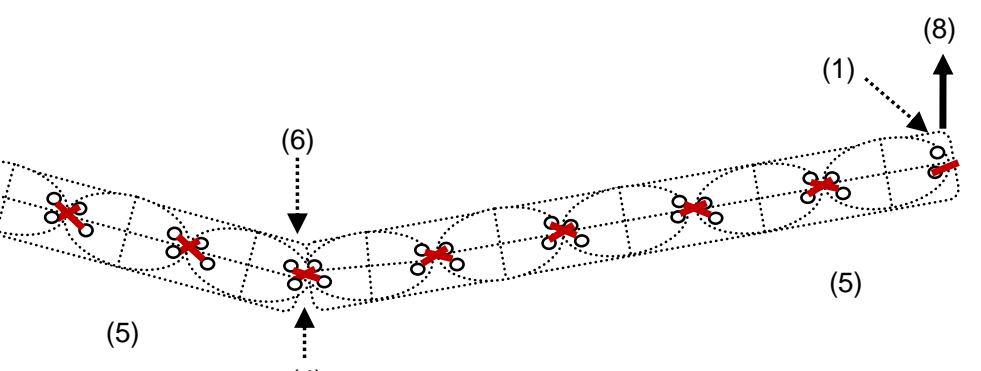

(4)

Existing void b/w

Existing void $\mathrm{b} / \mathrm{w}$
elongated atoms

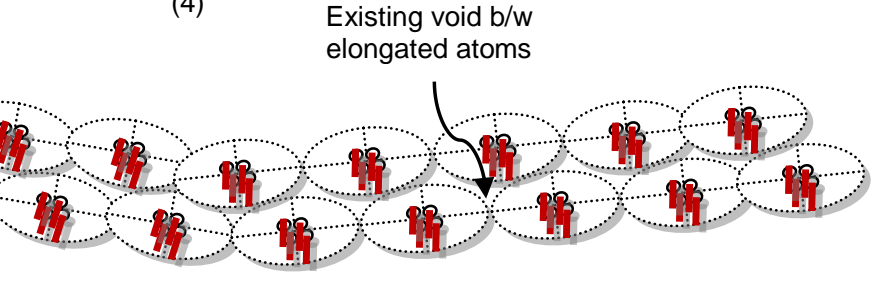

Figure 4: (a) electronically decreasing level solution surface (an inclined surface dealing with combined and controlled forces in immersing format), (b) adjacent-wise assembling of structures of smooth elements (1) non-assembling end, (2) energy knot of unfilled state, (3) energy knot of filled state, (4) common point of assembling structures of smooth elements, (5) structures of smooth elements coming from opposite sides to assemble at a common point, (6) assembling point of structures of smooth elements, (7) exertion of force in grounded format and (8) exertion of force in space format and (c) lateralwise adhering of shaped mono layers through their structures of smooth elements 
In assembling two structures of smooth elements adjacently, electron at the tip of elongated atom forming the assembling end of structure of smooth element rightly experiences the force through emptiness of unfilled energy knot at the tip of elongated atom forming the assembling end of structure of smooth element approaching from the opposite side. The same is the case with other structure of smooth element coming from the opposite side where an electron at the tip of elongated atom rightly experiences the force through emptiness of unfilled energy knot at the tip of elongated atom of oppositely sided structure of smooth element. As shown in Figure 4 (b), structures of smooth elements assemble, where clamping of unfilled energy knots to electrons of tips is in a crossed shape nucleating mono layer (of particle adjacent-wise).

Adhering lateral-wise structures of smooth elements in two shaped mono layers is shown hypothetically in Figure 4 (c). In lateral-wise adherence of shaped mono layers, their structures of smooth elements adhered in lateral manner. Electrons of zeroth rings in elongated atoms of structures of smooth elements belonging to upper shaped mono layer trap in the existing voids between elongated atoms of structures of smooth elements belonging to underneath shaped mono layer. (Electrons of zeroth rings in elongated atoms of structures of smooth elements belonging to underneath shaped mono layer trap in the existing voids between elongated atoms of structures of smooth elements belonging to upper shaped mono layer). So, to nucleate a particle of geometrical shape laterally, structures of smooth elements adjust at a common point to adhere laterally. Adherence of two shaped mono layers is in the interval of only a few microseconds, where upper shaped mono layer deals with exceeded gravity and underneath shaped mono layer deals with exceeded levity [13].

In the upper shaped mono layers dealing with localized gravity, a force exerting to electrons of elongated atoms downward exceeded to force exerting upward. In the underneath shaped mono layers dealing with localized levity, a force exerting to electrons of elongated atoms upward exceeded to force exerting downward. So, rightly positioned two shaped mono layers adhere in the lateral manner (as estimated in Figure 4c). On adhering structures of smooth elements (in upper shaped mono layer) to structures of smooth elements (in lower shaped mono layer), exertion of surface forces to laterally orientated electrons vanish. Now, a nanoparticle or particle deals with the 
forces of medium and own drawn poles. A detailed study on forcing or forced exertions in amalgamation of nanoparticle and particles inside the solution has been given elsewhere [29].

Due to the packing of tiny-shaped particles at high degree angles, structures of smooth elements assemble to nucleate MD shaped particles. In the assembling structures of smooth elements, tiny-shaped particles come from the different regions of zone having the same trend of exerting forces (east-west poles and near regions of east-west poles at solution surface). In the nucleation of a particle of 1D shape, packing of tiny-shaped particles and assembling structures of smooth elements are at low degree angles. In the assembling structures of smooth elements, tiny-shaped particles come from the different regions of a zone nearly keep the same nature of exerting forces. So, a particle of 1D shape is nucleated due to the arrival of tiny-shaped particles from a zone consisting of regions rearward to north-pole (and near regions rearward to north-pole) at solution surface. A particle MD shape is nucleated due to the arrival of tiny-shaped particles from a zone consisting of east-west regions (and near east-west regions) at solution surface. In addition to nucleation of particle of MD shape or $1 D$ shape, assembling structures of smooth elements for growth is also due to adjacent orientation of electrons and energy knots.

In the gold atom, both filled state electrons of the outer ring remain just below the east-west poles along the central horizontal line (to have the solid behavior in gold element). Possibly, one electron is in the state near the west-pole of its atom and one electron is in the state near the east-pole of its atom. In the central regions of solution surface, forces deal with a bit perturbed exertion for electrons, so they disturb the order of the 'structures of smooth elements' to a slight extent. So, they misalign the orientations of electrons to a slight extent in their clamped energy knots. However, overall shape of different particles remains geometrical. The individual dynamics of particles can arise due to medium interactions that could deteriorate the structures of smooth elements to some extent, too. This is mainly in the case of a pentagon-shaped particle. The associated defects with the shape are known as twin defects. So, forces remained slightly in disturbance at nucleation stage of particles due to the reflexes of near and far counterparts of the medium. In Figure 1 ( A, B, C, D, E, F \& G), spotted 
lines (and dots) in the SAPR patterns of rod and bar-shaped particles also indicate slightly disturbed orientation of electrons. Thus, upper and lower quadrants of solution surface belonging to rear sides of north-pole experienced the forces for electrons of elongated atoms in a bit uneven manner. A force of south-pole also influences to southsided tips of electrons (in elongated atoms of structures of smooth elements) at less pronounced level.

In the case of particles of 1D shapes, printed intensity of forced energy in reflected photons is in the form of lines. This is because of the less intensive elongation of atoms forming structures of smooth elements. Less intensive elongation of atoms takes place because of perpendicularly entering force (of north-pole) to solution surface. A force entering from the north-pole also combines with the forces of east-west poles. So, their exertions to electrons of less elongated atoms become slightly more disturbed. Therefore, lower elongation rate of atoms belonging to tiny-shaped particles nucleated a particle of 1D shape such as rod or bar, where a force along the north-pole is intervened at extensive level. A force along the south-pole (from the downward side to solution surface) is also intervened. In the regions of solution surface, where north-pole force is not intervened at exceeded level, particles of MD shapes such as hexagon, pentagon or triangle are nucleated.

More elongation of atoms of tiny-shaped particles develops a particle of MD shape, where their electrons belonging to the zeroth ring in elongated atoms are yet in the lateral orientation. So, featured photons interact with north-sided tips of electrons having lateral orientation in more elongated atoms of structures of smooth element. Photons are reflected to print forcing energy at fixed relative positions in the pattern. Printed intensities of reflected photons in the patterns of each particle having MD shape are in the form of dots. Here, atoms (of tiny-shaped particles) form structures of smooth elements, which experience force either in surface format along the east-west $\left(0^{\circ}\right.$ and $\sim 180^{\circ}$ or $\sim 180^{\circ}$ and $\left.\sim 360^{\circ}\right)$, or in the near regions of east-west $\left(0^{\circ}\right.$ to $\sim 74^{\circ}, \sim 106^{\circ}$ to $\sim 180^{\circ}$, $\sim 180^{\circ}$ to $\sim 254^{\circ}$ and $\sim 286^{\circ}$ to $\sim 360^{\circ}$ ).

Printed intensity of reflected photons in the patterns of particles having 1D shape is in the form of lines. Electrons of the outer rings also undertake adjacent orientations at less degree under the less stretching of clamping energy knots in addition to 
electrons of zeroth rings of elongated atoms. Tiny-shaped particles developing particles of 1D shape do not fully experience the forces of surface and a force of north-pole is also intervened. From the bottom side of the solution surface, a force of south-pole opposite to the north-pole also contributes. So, less elongation of atoms of tiny-shaped particles takes place while developing a particle of 1D shape. Electrons belonging to the zeroth ring of elongated atoms remained in the lateral orientations. Featured photons interacted with north-sided tips of electrons in less elongated atoms of front surface of particle having 1D shape. They are reflected to print the forced energy in the pattern at fixed relative positions in the form of lines. Here, the exerting force at electron level (in atoms) is pronounced less as their structures of smooth elements (in tiny-shaped particles) remain in the near regions rearward to north-pole at solution surface (in upper side of solution $\sim 74^{\circ}$ to $\sim 88^{\circ}$ and $\sim 92^{\circ}$ to $\sim 106^{\circ}$ and in lower side of solution $\sim 254^{\circ}$ to $\sim 268^{\circ}$ and $\sim 272^{\circ}$ to $\sim 286^{\circ}$ ).

Due to the nature of fundamental forces exerting to gold atoms at solution surface, assembling structures of smooth elements at orientation lower than $\sim 5^{\circ}$ is not viable. In Figure 1 ( $A, B, C, D, E, F \& G$ ), none of the rod or bar-shaped particles show orientation of assembling (adjacent-wise) structures of smooth elements lower than $\sim 5^{\circ}$. This deviation of $\sim 5^{\circ}$ becomes zero in atoms of semi-solid behavior [6]. At solution surface, regions belonging to rear sides of north-pole forming angles $\sim 88^{\circ}$ to $\sim 92^{\circ}$ and $\sim 268^{\circ}$ to $\sim 272^{\circ}$ (as shown in Figure 5 ) deal with lesser influence of forces exerted to electrons of gold atoms. Level of forced exertion to electrons of gold atoms increases while moving left and right to rear sides of north-pole at solution surface; both in upper quadrants and lower quadrants. Thus, exertion level of forces becomes the maximum along east-west poles, such as $0^{\circ}$ and $\sim 180^{\circ}$ or $\sim 180^{\circ}$ and $\sim 360^{\circ}$. In addition to two categorized zones of developing tiny-shaped particles, an overlapped zone (under the addition of $\sim 15^{\circ}$ angle) is also projected by double-lined arrows in Figure 5, where particles of partially overlapped features (in 1D and MD shapes) developed. (Particles shown in Figure 1a \& b appear from the surface in slightly overlapped or mixed featured shapes, but they still show shapes of rod or bar.) In Figure 5, outer and inner sides of tip of an electron in elongated atom are also labeled. 


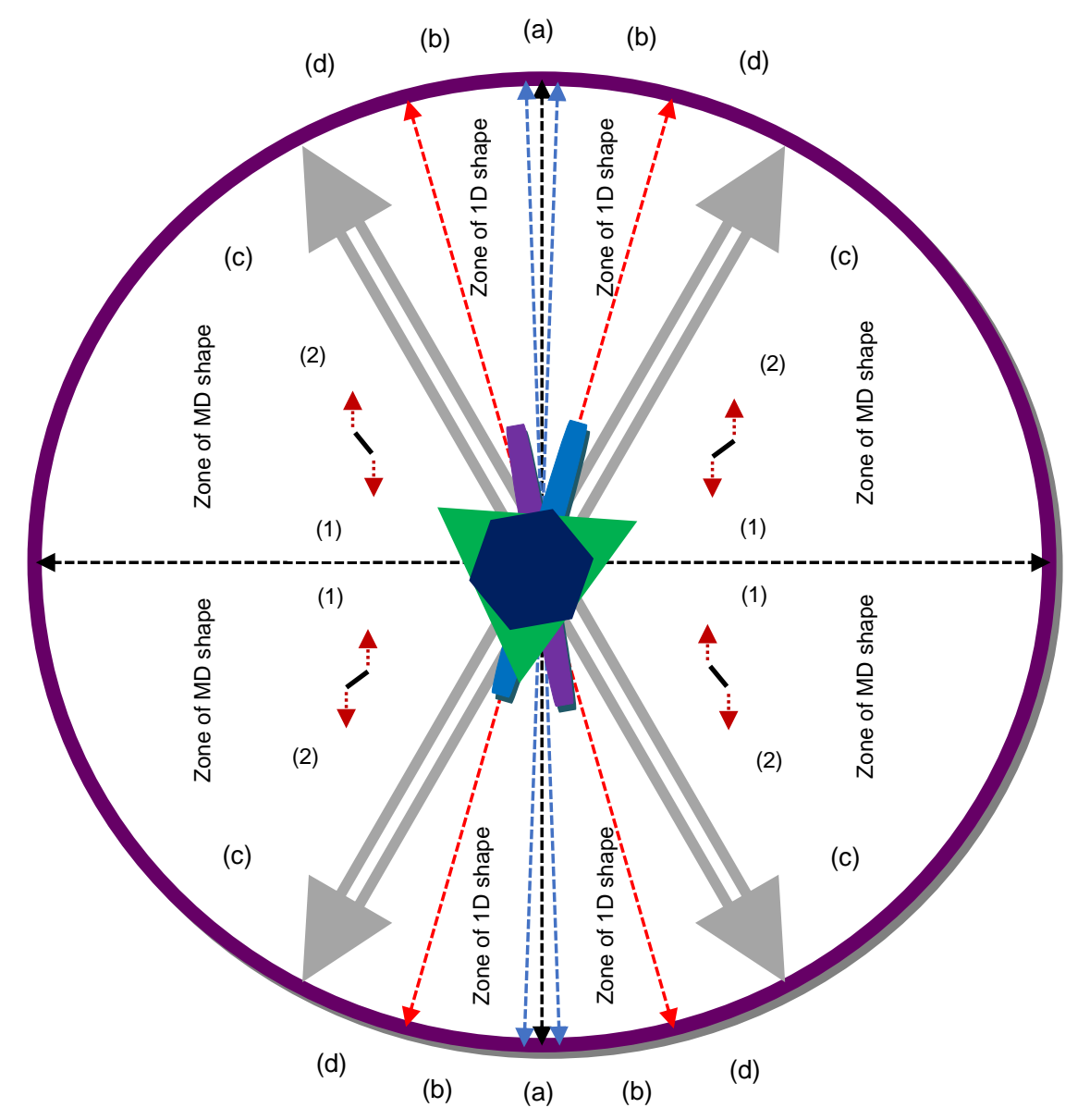

Figure 5: (a) different regions of zone dealing with negligible exertion of force to gold atoms (at electron level) at solution surface, different regions of zone of tiny-shaped particles nucleating particles of (b) 1D shapes, different regions of zone of tiny-shaped particles nucleating particles of (c) MD shapes, and different regions of mixed zone of tiny-shaped particles nucleating particles of (d) overlapped features (in 1D shapes and MD shapes); (1) inner and (2) outer tips of electrons in elongated atoms

In a high resolution microscopy analysis, when a featured photon interacted with north-sided tip of a laterally orientated electron belonging to zeroth ring of elongated atom forming the front surface of 'structure of smooth element' in front-sided mono layer of MD particle, the element of its force is reverted and that photon starts travelling to opposite side from the opposite end by forming the same angle at which it interacted. So when a featured photon interacted with north-sided tip of a laterally orientated electron, it shifted the phase by reverting the force. The reflection of photon is on the opposite side of normal line drawn to the centre of electron. This photon reflection occurs because of eccentricity of deviation. Thus, the law of reflection is related to reflection of photons (light) when interacted with north-sided tips of laterally orientated 
clamped energy knot electrons of solid behavior atoms. Here, incidence angle remains equal to reflection angle, which is in accordance with the original spirit of law. While interaction of featured photon with north-sided tip of a laterally orientated electron, electron remains held by clamping energy knot, where a recoverable infinitesimal displacement works to restore its position. The SAPR pattern of a particle under structural analysis is captured only by one shot of featured photons released from the source as per built-in flash, where they mostly do not interfere with photons just reflecting to print forced energy (intensities) in a pattern.

An electron clamped by energy knot in elongated atom having fixed orientation along north-pole is shown in Figure 6 (a). When a photon interacts with the tip of fixedpositioned electron, it bounces back as shown in Figure 6 (b). On interacting photon with the fixed-positioned electron from the advancing side, that photon also changes the position of side in addition to phase shift as displayed in Figure 6 (b). In Figure 6 (c), when a photon interacted from the right side with the fixed-positioned electron of an elongated atom, the reversion mechanism of the photon obeys the same principle as shown in Figure 6 (b). The incidence angle of an overt photon (short length photon) is approx. within $75^{\circ}$ to $85^{\circ}\left(75^{\circ} \leq \theta \leq 85^{\circ}\right)$ from either side of interaction with the fixedpositioned electron, so similar is the case with reflection angle. The angle of formation is from the horizontal line drawn through the centre of elongated atom or mid of energy knot clamped electron. When a photon interacts with the side of electron by forming an angle less than $70^{\circ}$ (ideally), it mainly converts into heat energy and when it interacts with the tip of electron by forming an angle $90^{\circ}$ (ideally), it mainly converts into pieces of bit energy [5]. On suitable interaction, the impact of fixed-positioned clamped energy knot electron reverses the force of reflected photon, where energy wrapping to force is affected as shown in different color in Figure 6 (b) and (c). In the reflection of photon, energy wrapping force governs its levitational mode. However, in the incidence of photon, energy wrapping force governs its gravitational mode. 


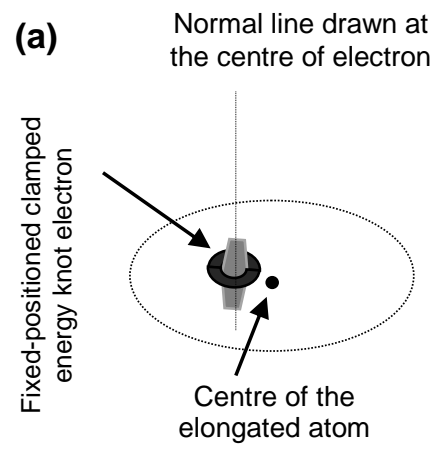

(b) Reflected photon

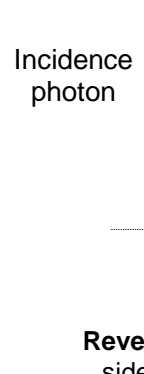

(c)

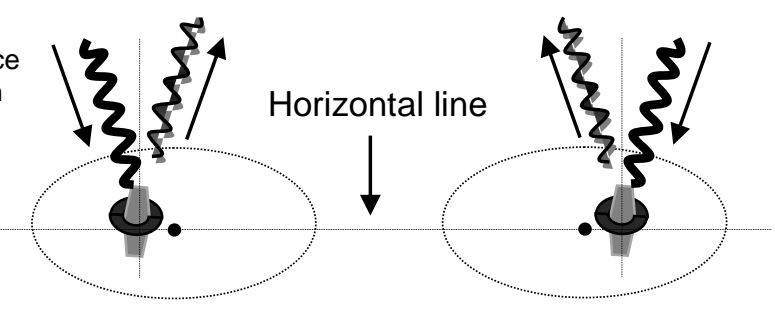

Reversion Mechanism of Photon: Photon interaction with northsided tip of laterally orientated electron of elongated gold atom

Figure 6: (a) clamped energy knot (fixed-positioned) electron of elongated atom (in front-sided mono

layer of 1D or MD shape), (b) reversion mechanism of photon when clamped energy knot (fixedpositioned) electron is on left side to the centre of elongated atom and (c) reversion mechanism of photon when clamped energy knot (fixed-positioned) electron is on right side to the centre of elongated atom

Electrons, on splitting of their inert gas atoms, carry chasing photons for impinging on the underneath atom to either elongate it or deform it [4]. So, electrons impinged under gained forced energy of carrying/chasing photons. However, featured photon is related to overt photon, which (as per set pace of their releasing) interacted with the north-sided tip of clamped energy knot electron of elongated atom. This way, photons are released from the built-in source (of analysis technique) without chasing electrons of splitting inert gas atoms. Photons are generated under the execution of electron dynamics in neutral state silicon atoms, where they work as a photonic current under certain arrangement [5]. This becomes clear from the SAPR patterns of 1D and MD shapes of gold particles. The selected area patterns shown against their particles in Figures 1 and 2 are perhaps the most conclusive evidences and proofs of photons reflection (rather than electrons diffraction). The spotting (printing) of intensity spots in the SAPR patterns of different geometrical shapes also reveals that a levitating force comes from the reversion mechanism of photons. Featured photons interact with laterally orientated electrons of atoms belonging to front surface structures of smooth elements resulting in the reversal of the elements of their force.

A photon, a travelling photon and a travelled photon are related to a force and energy relationship, a forcing energy and a forced energy respectively. A structure of adequate alignment regulates the forcing energy of a propagating photon through it. This is the case in both particles of 1D and MD shapes. A distorted structure disturbs the propagation of photons because of having misaligned electrons. However, 
interaction of featured photons with north-sided tips of laterally-orientated electrons (of front surface of $1 \mathrm{D}$ and MD shapes) results in printing their forced energy in patterns, and thus providing the precise identification of structure of elongated atoms particles.

Many studies discussed synthesis of nanoparticles and particles, where mechanism of development was mainly addressed in the light of electrostatic, hydrodynamic and electrodynamic interactions. These studies are based on the concepts of charges on atoms. Colloidal atoms assembled in 2D configuration under the application of thermoelectric field but incorporation of that field into an optical radiation force has resulted in the reconfiguration of the $2 \mathrm{D}$ colloidal matter configuration into $3 \mathrm{D}$ [30]. Different named forces other than the fundamental forces were being considered to comprehend the complex structural hierarchies and gadgets of colloidal nanoparticles [31]. Another study discussing the optical fields to drive the colloidal systems has been demonstrated [32]. The synthesis of colloidal nanoparticles has been reported recently in which the science of electrodynamic and electrostatic interactions is revisited, where entropic behavior of their chain switches to anisotropic under a tunable laser power [33].

Dynamics are considered to explain structure in terms of entropy and geometry in many studies, and some of them are referred here [18, 28, 32, 33]. Nonetheless, dynamics are expected to be discussed with usefulness when the intrinsic nature of an atom is known. Details for atoms of different elements to sufficient extents have been given elsewhere [1-10]. These lead to explain the dynamics with relevance to method or process and its input source of power, precursor and processing conditions. Hence, colloidal atoms first influence (inspire) the study of attained dynamics following their electron dynamics, which is purely related to intrinsic nature. Second, overall processing conditions regulate the dynamics in terms of extrinsic behavior of atoms and particles, where input sources of force and energy or energy and force (power) play a vital role. They combine to develop the structure of colloidal atoms. Since long, it has been described by the probe of stimulating thoughts that prior to assembling a tiny-sized particle, a study of dynamics for individual formation of a tiny particle is essential [28]. Our study worked out this well. Further, our study extends those unprecedented thoughts by discussing the dynamics of atoms in the formation of tiny particles, nanoparticles and particles. So, the present work establishes an effort towards the 
accomplishment of the reality (stated in ref. 27) that atoms and molecules will become future materials on understanding the assembling of building blocks for larger particles.

The role of dynamics becomes perceptible when tiny grains of carbon films are synthesized in microwave-based vapor deposition technique, where carbon atoms related to different tiny grains exhibited several equilibrium states [34]. Atomically precise nanoparticles are extremely beneficial to determine the total structure and fundamental science [35]. Doping or alloying of heterometals to gold nanoparticles can preserve the structure providing them extraordinary stability [36]. Several important questions regarding the study of colloidal nanoparticles are still needed to explore [37]. A recent study discloses an important role of orientation force to describe the functioning of structure [38].

Here, when transitional behavior atoms are shaped in compact assembly at solution surface, they are related to a "monolayer". However, when elongated atoms are shaped in tiny-shaped particles or particles of 1D and MD shapes, they are related to a 'mono layer'. Generally, they are related to a 'shaped mono layer'. An elongated atom is related to adjacent orientation of its electrons. In line with this, different theories related to Ostwald ripening (monomer to monomer addition), crystallography, etc. should be revisited in formation and description of nanoparticles and particles. As tiny particles and their atoms obey the chemio-physio mechanism simultaneously to nucleate particles, their science explains both physical and chemical mechanisms. Hence, anisotropy or geometry of particle is controlled by binding atoms during elongating, adjacent-wise assembling of structures of smooth elements (of shaped mono layers) and lateral-wise adhering of structures of smooth elements (of shaped mono layers), where fundamental forces exert in an extremely diligent fashion.

\section{Conclusion}

This study presents an understanding of science at atomic, nano and micro levels. Nature of forces exerting to electrons of gold atoms depends on the position of their developing tiny-shaped particles (at electronically flat solution surface). At solution surface, forces exerting to atoms of tiny-shaped particles in east-west regions are 
greater in influence than forces exerting to atoms of tiny-shaped particles in regions rearward to the north-pole.

In zones categorized in particles having 1D shapes and MD shapes, arrays of different tiny-shaped particles converted into structures of smooth elements, which show different width and inter-spacing distance. On assembling and adhering structures of smooth elements, different particles of $1 \mathrm{D}$ and MD shapes develop. In particles of rod or bar shape, photonic propagation is along the 'direction of one established side' (unidirection), so they plot dimensional regularity in 1D shapes. In particles of triangular or hexagonal shape, photonic propagation is along the direction of three established sides or six established sides, so they plot dimensional regularity in their MD shapes.

Forces of all four poles exert to electrons (of elongated atoms forming structures of smooth elements) at electronically decreasing level solution surface. Thus, an electronically decreasing level solution surface is related to that inclined surface, where forces under simultaneous actions exert to a structure of smooth element. At electronically decreasing level solution surface, a structure of smooth element assembles due to exerting forces in the immersing format. In immersing format, on one side, an exerting force in surface format along the assembling end of structure of smooth element becomes greater than the exerting force in surface format along the non-assembling end. In immersing format, on the other side, exerting forces to structure of smooth element in space format and grounded format compete for equivalence.

Tiny-shaped particles advance from different regions of solution surface to assemble their structures of smooth elements at a common point under the exertion of forces in immersing format. Assembling of structures of smooth elements in adjacentwise nucleate and grow shaped mono layers to develop a nanoparticle or particle of geometrical shape. To nucleate a particle of geometrical shape adjacently, structures of smooth elements assemble through orientated electrons and unfilled energy knots (of elongated atoms) available at their tips. Depending on the orientation of assembling (adjacent-wise) structures of smooth elements, they form a shaped mono layer of a different geometry.

To nucleate a particle of geometrical shape laterally, a developing shaped mono layer adheres to underneath shaped mono layer developed firstly. By occupying the 
centre of concave meniscus at solution surface, structures of smooth elements of both shaped mono layers adhere laterally [13]. In the lateral-wise adherence of two shaped mono layers, laterally orientated electrons trap (or fix) in the existing voids of elongated atoms (forming structures of smooth elements) on reciprocal basis. Here, laterally orientated electrons of elongated atoms in upper shaped mono layer experience exceeded gravity, so they move a bit downward and laterally orientated electrons of elongated atoms in underneath shaped mono layer experience exceeded levity, so they move a bit upward. In developing nanoparticle or particle of geometrical shape, adherence of shaped mono layers obeys the same mechanism to extend laterally.

Orientations of electrons in clamped energy knots vary the force behavior for their positional atoms in different tiny-shaped particles. A force exerting to electron influence it, but that force cannot influence the clamping energy knot of that electron. So, changing the manner of energy knot clamping electron varies the level and trend of force exerting for it. At common point of assembling structures of smooth elements, tinyshaped particles developing rod and bar-shaped particles deal with exerted force in a bit perturbation.

In the selected area photon reflection (SAPR) pattern of a particle, intensity spots when printed in fixed distant parallel dots, they signify the structure of more elongated atoms, which is in the case of its MD shape. Intensity spots, when printed in fixed distant parallel lines, they signify the structure of less elongated atoms, which is in the case of particle of 1D shape. In particles of 1D shapes, printed intensity spots in fixed distant parallel lines are due to reverted element of force in reflected photons. Here, not only are the laterally orientated electrons of zeroth rings in elongated atoms involved in reflecting photons, but the laterally orientated electrons of outer rings are also involved in reflecting photons. This is not the case in particles of MD shapes. In particles of MD shapes, photons print circular dots of fixed mid to mid distance (fixed distant parallel dots) on reflection. In MD shapes, atoms elongated more in arrays of tiny-shaped particles where mainly the electrons of zeroth rings remain laterally orientated.

A reflection of photon is due to the reverted element of its force. Interaction of photons with north-sided tips of laterally orientated electrons results in reverting the element of their forces. This sort of force also works as the source of levity in ordinary 
environment, where it does not print to show spot. On interacting featured photons with north-sided tips of laterally orientated electrons of front surface elongated atoms, they switch the phase to opposite side of drawn normal line, thus reverting the element of their forces. A photon reflects from the opposite side of normal line by keeping the same angle as in incidence. Exerting force to electrons in atoms while in surface format is because of the levitation factor. The source of levity is reversion mechanism of photon.

To explore the science of different phenomenon and mechanism for sustainable technologies and environment, bottom to top approaches are more viable. Science of different phenomenon and mechanism can also be explored when studying materials (at macro and micro levels) under the top to bottom approaches. The investigations discussed here give birth to a new era of science.

\section{Acknowledgements}

This study was funded by the National Science Council, now Ministry of Science and Technology, Taiwan (NSC-102-2811-M-032-008; 2013-2014). Authors wish to thank Mr. Chien-Jui Yeh for assisting in microscopic operation. The first author appreciates the support in miscellaneous matters from the Researchers of Professor Lin's group.

\section{References}

[1] Ali, M. Atomic Structure and Binding of Carbon Atoms. (2019), https://www.preprints.org/manuscript/201801.0036 (last version)

[2] Ali, M., Hamzah, E., Toff, M. R. M. Hard Coating Deposits: Incompatible Working Energy and Forced Behaviors of Gaseous and Solid Atoms. (2019), https://www.preprints.org/manuscript/201802.0040 (last version)

[3]. Ali, M. Structure Evolutions in Atoms of Those Elements Executing Confined Inter-State Electron Dynamics. (2020), http://arxiv.org/abs/1611.01255 (last version)

[4] Ali, M. Atoms of None of the Elements Ionize While Atoms of Inert Behavior Split by Photonic Current. (2020), http://arxiv.org/abs/1611.05392 (last version) 
[5] Ali, M. Heat and Photon Energy Phenomena: Dealing with Matter at Atomic and Electronic Level. (2017), https://www.preprints.org/manuscript/201701.0028 (last version)

[6] Ali, M. Atoms in Gas and Solid States and their Energy and Force Relationships under Transitional Behaviors. (2020), https://www.researchgate.net/publication/323723379

[7] Ali, M. Nanoparticles -Photons: Effective or Defective Nanomedicine. J. Nanomed. Res. 5 (6), 241-243 (2018).

[8] Ali, M., Lin, I $-\mathrm{N}$. Effects of the Electronic Structure, Phase Transitions and Localized Dynamics of Atoms in the Formation of Tiny Particles of Gold. (2020), http://arxiv.org/abs/1604.07144 (last version)

[9] Ali, M., Lin, I-N., Yeh, C. -J. Tapping Opportunity of Tiny-Shaped Particles and Role of Precursor in Developing Shaped Particles. NANO 13 (7) (2018) 1850073 (16 pages).

[10] Ali, M., Lin, I -N. Formation of tiny particles and their extended shapes: origin of physics and chemistry of materials. Appl. Nanosci. 9 (6) (2019) 1367-1382

[11] Ali, M., Lin, I $-\mathrm{N}$. Controlling morphology-structure of gold tiny particles, nanoparticles and particles at different pulse rates and pulse polarity. Adv. Nat. Sci.: Nanosci. Nanotechnol. 10 (2) (2019) 025015 (14 pp).

[12] Ali, M., Lin, I -N. Development of Gold Tiny Particles and Particles in Different Sizes at Varying Precursor Concentration. Adv. Nat. Sci.: Nanosci. Nanotechnol. 11 (1) (2020) 015006.

[13] Ali, M. Tiny-Shaped Particles Developing Mono Layer Shape Dealing with Localized Gravity at Solution Surface. (2020), http://arxiv.org/abs/1609.08047 (last version)

[14] Ali, M., Lin, I -N., Yeh, C. -J. Predictor Packing in Developing Unprecedented Shaped Colloidal Particles. NANO 13 (9) (2018) 1850109 (15 pages).

[15] Zhu, Y., Ramasse, Q. M., Brorson, M., Moses, P. G., Hansen, L. P., Kisielowski, C. F., Helveg, S. Visualizing the Stoichiometry of Industrial-Style Co-Mo-S Catalysts with Single-Atom Sensitivity. Angew. Chem. Int. Ed. 2014, 53, 1072310727. 
[16] Kisielowski, C. Observing Atoms at Work by Controlling Beam-Sample Interactions. Adv. Mater. 27, 5838-5844 (2015).

[17] Negishi, Y. et al. A Critical Size for Emergence of Nonbulk Electronic and Geometric Structures in Dodecanethiolate-Protected Au Clusters. J. Am. Chem. Soc. 137, 1206-1212 (2015).

[18] Manoharan, V. N. Colloidal matter: Packing, geometry, and entropy. Science 349, 1253751 (2015).

[19] Suzuki, Y., Cardone, G., Restrepo, D., Zavatteri, P. D., Baker, T. S., Tezcan, F. A. Self-assembly of coherently dynamic, auxetic, two-dimensional protein crystals. Nature 533, 369-373 (2016).

[20] Evers, C. H. J., Luiken, J. A., Bolhuis, P. G., Kegel, W. K. Self-assembly of microcapsules via colloidal bond hybridization and anisotropy. Nature 534, 364368 (2016).

[21] Epstein, I. R., Xu, B. Reaction-diffusion processes at the nano- and microscales. Nature Nanotech. 11, 312-319 (2016).

[22] Matsumoto, Y. Vascular bursts enhance permeability of tumour blood vessels and improve nanoparticle delivery. Nature Nanotech. 11, 533-538 (2016).

[23] Park, J., et al. 3D structure of individual nanocrystals in solution by electron microscopy. Science 349, 290-295 (2015).

[24] Jacobsson, D., et al. Interface dynamics and crystal phase switching in GaAs nanowires. Nature 531, 317-322 (2016).

[25] Tuma, T., Pantazi, A., Gallo, M. L., Eleftheriou, E. Stochastic phase-change neurons. Nature Nanotech. 11, 693-699 (2016).

[26] Rensberg, J., et al. Active Optical Metasurfaces Based on Defect-Engineered Phase-Transition Materials. Nano Lett. 16, 1050-1055 (2016).

[27] Glotzer, S. C., Solomon, M. J. Anisotropy of building blocks and their assembly into complex structures. Nature Mater. 6, 557-562 (2007).

[28] Link, S., El-Sayed, M. A. Shape and size dependence of radiative, nonradiative and photothermal properties of gold nanocrystals. Int. Rev. Phys. Chem. 19, 409453 (2000). 
[29] M. Ali, I -N. Lin. Forcing or Forced Exertions in Amalgamation of Nanoparticles and Particles inside the Solution. ChemRxiv. Preprint. (2020), https://doi.org/10.26434/chemrxiv.9823625 (last version)

[30] Lin, L., et al. Opto-thermophoretic assembly of colloidal matter. Sci. adv. 3, e1700458 (2017).

[31] Gargiulo, J., et al. Understanding and Reducing Photothermal Forces for the Fabrication of Au Nanoparticle Dimers by Optical Printing. Nano Lett. 17, 57475755 (2017)

[32] Figliozzi, P., et al. Driven optical matter: Dynamics of electrodynamically coupled nanoparticles in an optical ring vortex. Phys. Rev. E 95, 022604 (2017).

[33] Nan, F., Han, F., Scherer, N. F., Yan, Z. Dissipative Self-Assembly of Anisotropic Nanoparticle Chains with Combined Electrodynamic and Electrostatic Interactions. Adv. Mater. 30, 1803238 (2018).

[34] Ali, M., Lin, I -N. Phase transitions and critical phenomena of tiny grains carbon films synthesized in microwave-based vapor deposition system. Surf. Interface Anal. 51 (2019) 389-399.

[35] Jin, R., Qian, H., Wu, Z., Zhu, Y., Zhu, M., Mohanty, A., Garg, N. Size Focusing: A Methodology for Synthesizing Atomically Precise Gold Nanoclusters, J. Phys. Chem. Lett. 1 (2010) 2903-2910.

[36] Jin, R., Nobusada, K. Doping and alloying in atomically precise gold nanoparticles, Nano Research 7 (2014) 285-300.

[37] Cuffari, B. Does the Structure of Gold Nanoparticles Change with Different Dopants? Feb. 11, 2019; https://www.azonano.com/article.aspx?ArticlelD=5142

[38] Ali, M. Transition Energy, Orientation Force and Work Done in Transitional Behavior Atoms: Formulating New Principles in Thermodynamics. ChemRxiv. Preprint. (2020), https://doi.org/10.26434/chemrxiv.11553057 (last version) 
Nomenclatures/definitions:

Electronically flat solution surface - an electronically flat solution surface is a solution surface which is flat to the extent of an electron

Electronically decreasing level solution surface - a (circular) solution surface decreasing with the rate of depth of electron level inwardly to develop a concave meniscus

Elongated atom - an elongated atom is the atom in which electrons orientated along the east-west poles by aligning the lateral orientation into adjacent orientation

Deformed atom - a deformed atom is the atom in which electrons orientated along the north-south poles as well as orientated along the east-west poles

Structure of smooth element - a structure of smooth element is formed (develop) by the array of elongated atoms forming a shape like line

Immersing format - an electronically decreasing level solution surface deals with the exertion of forces in immersing format, where the force at electron level functions in a controlled manner along its east-pole, west-pole, north-pole and south-pole simultaneously

Clamped energy knots electrons - in atoms of different elements, electrons remain clamped by their clamping energy knots in original behavior as well as in transitional behavior; however, in elongated atom, clamped energy knots to electrons are unidirectionally stretched and in deformed atom, clamped energy knots to electrons undergo non-unidirectional stretching 


\section{Authors' biography:}

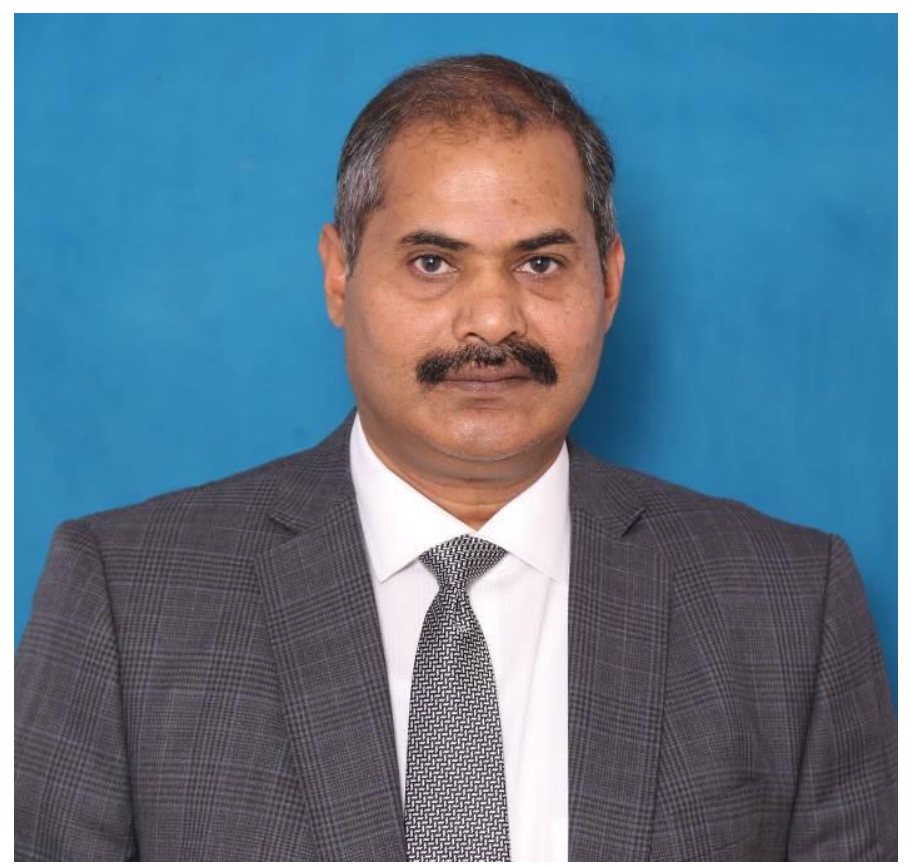

Mubarak Ali graduated from University of the Punjab with BSc (Phys \& Maths) in 1996 and MSc Materials Science with distinction from Bahauddin Zakariya University, Multan, Pakistan (1998); his thesis work completed at Quaid-i-Azam University Islamabad. He gained PhD in Mechanical Engineering from the Universiti Teknologi Malaysia under the award of Malaysian Technical Cooperation Programme (MTCP;2004-07) and postdoc in advanced surface technologies at Istanbul Technical University under the foreign fellowship of The Scientific and Technological Research Council of Turkey (TÜBITAK, 2010). He completed another postdoc in the field of nanotechnology at the Tamkang University Taipei (20132014) sponsored by National Science Council now M/o Science and Technology, Taiwan (R.O.C.). Presently, he is working as Assistant Professor on tenure track at COMSATS University Islamabad (previously known as COMSATS Institute of Information Technology), Islamabad, Pakistan (since May 2008) and prior to that worked as assistant director/deputy director at M/o Science \& Technology (Pakistan Council of Renewable Energy Technologies, Islamabad, 2000-2008). He was invited by Institute for Materials Research, Tohoku University, Japan to deliver scientific talk. He gave several scientific talks in various countries. His core area of research includes materials science, physics \& nanotechnology. He was also offered the merit scholarship for the PhD study by the Higher Education Commission, Government of Pakistan, but he did not avail himself of the opportunity. He also earned Diploma (in English language) and Certificate (in Japanese language) in 2000 and 2001 respectively, in part-time from the National University of Modern Languages, Islamabad. He is the author of several articles available at following links; https://scholar.google.com.pk/citations?hl=en\&user=UYjvhDwAAAA, https://www.researchgate.net/profile/Mubarak Ali5, https://www.mendeley.com/profiles/mubarak-ali7/, \& https://publons.com/researcher/2885742/mubarak-ali/publications/ 


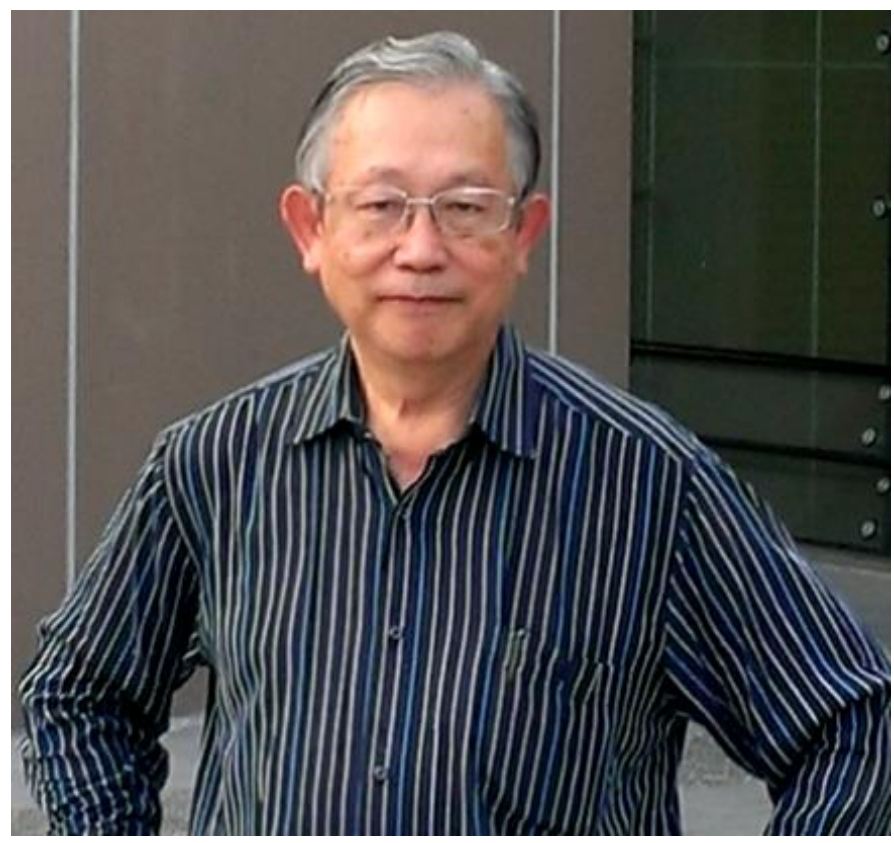

His Excellency, I-Nan Lin, worked as a Senior Professor for several years at Tamkang University, Taiwan, and now Professor Emeritus. His Excellency received the bachelor's degree in physics from National Taiwan Normal University, Taiwan, M.S. from National Tsing-Hua University, Taiwan, and the Ph.D. degree in Materials Science from U. C. Berkeley in 1979, U.S.A. His Excellency, worked as a senior researcher in Materials Science Centre in TsingHua University for several years and a faculty member as senior professor in Department of Physics, Tamkang University. Professor Lin published several hundred referred journal publications and held distinctive position in university. Professor Lin supervised several PhD and Postdoc candidates around the world. His Excellency remained researching in the areas of developing high conductivity diamond films, composite materials and the deeprooted studies of the transmission microscopy of materials. 